\title{
Wavefront Sensing using a Multi-Object Spectrograph (NIRSpec)
}

\author{
Bruce H. Dean, ${ }^{a}$ Rene Boucarut, ${ }^{b}$ Theo Hadjimichael, ${ }^{\mathrm{c}}$ Scott Smith ${ }^{\mathrm{d}}$ \\ Optics Branch, NASA Goddard Space Flight Center, Greenbelt, MD 20771
}

\begin{abstract}
An analysis is presented that illustrates how the James Webb Space Telescope (JWST) fine-phasing process can be carried out using the Near-Infrared Spectrograph (NIRSpec) data collected at the science focal plane. The analysis considers a multi-plane diffraction model which properly accounts for the microshutter diffractive element placed at the first relay position of the spectrograph. Wavefront sensing results are presented based on data collected from the NASA Goddard Microshutter Testbed.
\end{abstract}

Keywords: wavefront sensing, phase retrieval, focus diversity, MEMs device, James Webb Space Telescope

\section{Introduction}

The JWST ${ }^{1}$ (James Webb Space Telescope) is one of NASA's great observatories of the Origins program ${ }^{2}$ and is scheduled to launch at the beginning of the next decade. The JWST will incorporate a multi-object spectrograph, or NIRSpec, ${ }^{3}$ the Near Infrared Spectrograph, as one of the primary science instruments on-board the spacecraft. As such, the NIRSpec instrument will utilize a portion of the science focal plane, with $\lambda$ in the range: 0.6 to $5 \mu$, to carry out investigations on galaxy formation, clustering, molecular structure, star formation, active galactic nuclei, and young stellar clusters.

The JWST will be constructed using advanced optical technology and extremely lightweight mirrors. ${ }^{4}$ The primary mirror is a 6.5 meter diameter 18 segment hexagonal array. The figure quality should be diffraction limited at $2 \mu$, operate at $30-60 \mathrm{~K}$, and have an areal density of less than $15 \mathrm{~kg} / \mathrm{m}^{2}$. As a result, the JWST commissioning phase and periodic maintenance/optical correction will utilize image-based wavefront sensing and control technology to align the mirror segments and minimize figure error as well as position the secondary mirror of the 3-mirror anastigmat design. The WFS method specified for JWST is image-based in the sense that point source images (or other known object) are collected to recover optical phase information. The primary camera for this function is NIRCam. ${ }^{5}$ An important risk mitigation strategy for the JWST design is based on engineering redundancy for observatory functions. Therefore, an obvious redundancy to consider is the WFS process itself which logically implies the use of multiple cameras for WFS. In addition, it has been noted that imaging performance over the JWST FOV can be better compensated by using simultaneous WFS results from

\footnotetext{
${ }^{a}$ For additional information send correspondence to: bruce.dean@ nasa.gov

brene.boucarut@nasa.gov

c thadjimichael@swales.com

d jeffrey.s.smith@nasa.gov
} 
multiple cameras using a Kalman filter approach. ${ }^{6}$ Degradation in optical quality due to WFS and control at a single field point has been considered in. ${ }^{7}$

To address the concern for sufficient WFS redundancy as well as WFS and control induced anisoplanatism - one possibility is to augment NIRCam WFS data using focal plane images collected by NIRSpec (while operating in its camera imaging mode). However, NIRSpec WFS is complicated by the fact that the spectrograph design employs a microshutter ${ }^{8}$ array at the first relay position of the spectrograph. As a result, diversity defocus data collected at the NIRSpec focal plane will be imprinted with a rectangular diffraction pattern induced by the microshutter array light shield. The subject of this paper is a discussion of WFS results obtained by processing these diversity defocus images using an image-based WFS algorithm. Modeling comparisons are also made utilizing multi-plane diffraction techniques which motivates the use of a simple overlay of the MS light shield upon the defocused intensity data. In Section 2, the microshutter arrays and GSFC Microshutter Testbed are discussed. Section 3 describes a multi-plane forward diffraction model that is used to calculate images for comparison to the MTB data. Image-based WFS is discussed in Section 4 the WFS results are presented. Conclusions are discussed in Section 5.

\section{Microshutter Arrays and Testbed Optical Design}

The microshutter array ${ }^{8}$ is a field programmable micro-electromechanical device that enables the NIRSpec instrument to select hundreds of different objects in a single field of view. Microshutters are an alternative to the micro-mirror ${ }^{9}$ systems which cannot meet JWST requirements for image quality, spectral coverage or contrast. Figure 1 shows a scanning microscope image of a portion of the microshutter array under development at the NASA Goddard Space Flight Center.

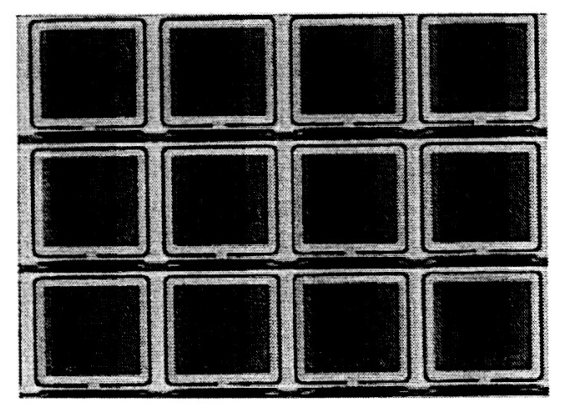

Figure 1. Scanning microscope image of a portion of the microshutters array.

A microshutter array consists of about $1000 \times 500$ microshutters, $100 \mu$ center-to-center and about $90 \mu$ wide. NIRSpec will employ a $100 \mu \times 200 \mu$ (spectral x spatial direction) array. All shutters are slightly magnetized and are opened by scanning a permanent magnet over the array. Selected apertures are held open electrostatically by applying a voltage difference between the shutter and an electrode on the wall. Following the single passing of the permanent magnet, the resilience of the hinges flips the remaining shutters closed. Additional refrences and information on the microshutter arrays can be found at http://bram.gsfc.nasa.gov/ms_webpage/ms_main.html. 
The MTB is a resource located at the GSFC and was designed to investigate and test the electro-mechanical operation of the shutters as well as electro-optical characteristics such as transmission and light leakage around closed shutters at infrared wavelengths. Early modeling efforts ${ }^{10}$ have focused on the electro-optical properties of the arrays.

The MTB has the following layout as labeled in Figure 2: a tungsten point-source is filtered at $0.633 \mu$ (and $2 \mu$ ) and then collimated by an off-axis parabola. The beam is then stopped by an iris which controls the plate scale at the microshutter array. This is variable to $F / 15$ (the fastest). Lens assembly one is an IR/visible triplet which focuses the beam to the microshutter. $\mathrm{CaF} 2$ windows are used as windows to a dewar which houses the microshutter array. The beam is then re-collimated to a $2^{\text {nd }}$ stop and then focused using a $2^{\text {nd }}$ off-axis parabola. The testbed setup includes both an Apogee CCD with $13 \mu$ pixels as and an Indigo Merlin 1.5 - 5.0 $\mu$ waveband InSb infrared Camera. The InSb array size is $320 \times 256$ with a $30 \mu$ pixel size.

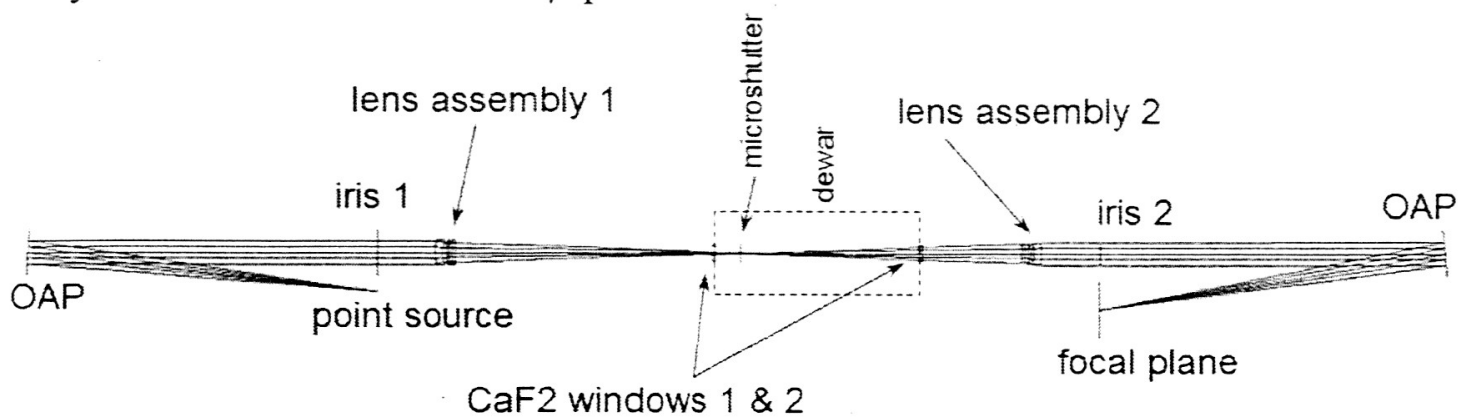

Figure 2. MTB Optical Design.

For comparison with the MTB layout, Figure 3 shows a MOS design in paraxial form. The point source at the MTB is analogous the input beam of the MOS design shown to the far left of Figure 3.

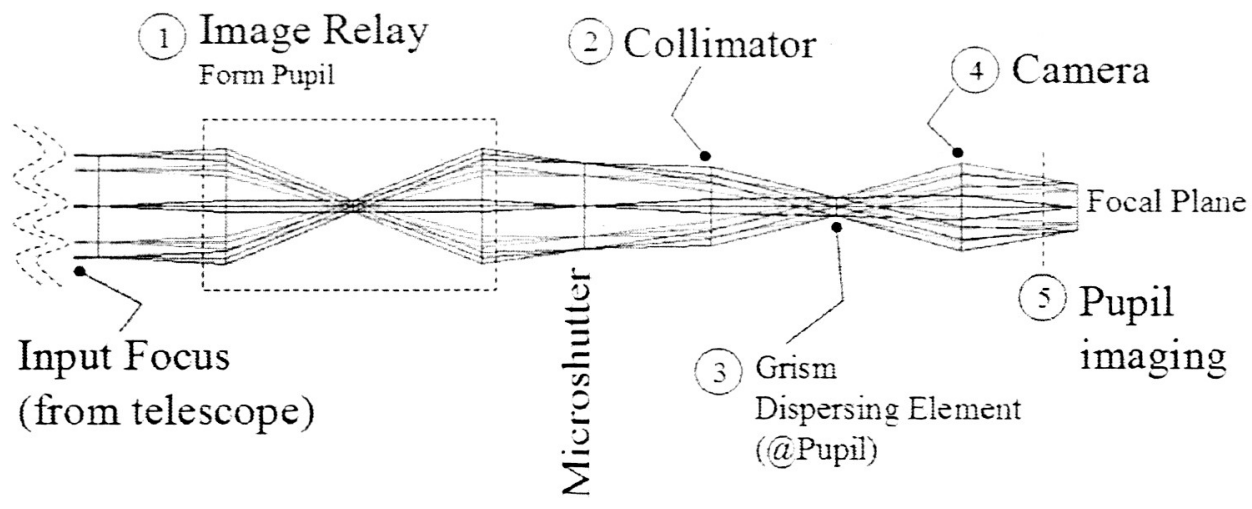

Figure 3. MOS in Paraxial Form.

The off-axis parabola of the MTB collimates to pupil space and then lens assembly 1 focuses the beam to the microshutter (labeled as (1) in Figure 3 ). The beam is recollimated using lens assembly 1 (labeled as (2) in Figure 3) and then the final off-axis parabola of the MTB serves as the camera lens labeled as (4). As a result, these is direct 
correspondence between the MTB and a MOS design and therefore we expect some correspondence in the WFS data. For the WFS data, the IRIS-1 was at about 0.6" to make the beam approximately F/48 at the microshutter. This is then relayed to F/51 at the detector to give a slightly oversampled PSF by a factor of 1.25 .

\section{Multi-Plane Diffraction Model and MTB Data}

Focus-diverse WFS requires intensity data collected at a defocused image plane. For NIRSpec, there are two options for introducing diversity defocus. The advantages and disadvantages to each are summarized in Table 1.

Table 1. Options for implementing diversity defocus

\begin{tabular}{lll}
\hline $\begin{array}{l}\text { Diversity } \\
\text { Defocus }\end{array}$ & Secondary Mirror Motion & NIRSpec Cam \\
\hline Advantage & (1) Common path diversity & (1) Secondary Mirror \\
& (2) Large defocus possible & Stationary \\
\hline Disadvantage & (1) Sec Mirror Motion; & (1) too small defocus ? \\
& (2) Diffractive shutter or & (2) camera movement \\
& slit & $\begin{array}{l}\text { (3) non-common path } \\
\text { diversity }\end{array}$ \\
\hline
\end{tabular}

The first option involves translating the secondary mirror along its optical axis. This has an important advantage in that all instruments of the ISIM science module will "see" diversity defocus and therefore WFS data can be collected in multiple cameras simultaneously. As a result, defocus consistency is easier to achieve since all cameras will receive common path defocus diversity. In addition, larger defocus values may be realized quite easily using secondary mirror motion. A disadvantage though is that defocus is introduced ahead of the MSA which has the consequence of introducing a rectangular grid structure onto the defocused image. However, as we show in this investigation, this artifact does not seriously degrade WFS performance. Another disadvantage is associated with the risk involved in moving such a major optical element of the OTE. Should the secondary mirror become stuck or frozen in its defocused position, the observatory will likely be rendered in-operable.

The second option involves translating the NIRSpec imaging camera directly along the chief-ray optical axis. But with a limited range of motion it may not be possible to obtain a sufficient amount of defocus to perform accurate WFS. Diversity selection for imagebased WFS has been previously analyzed based on the spatial frequency content of the input aberration. ${ }^{11}$ Additional analysis on accuracy vs defocus has also been presented. ${ }^{12}$ In addition, the problem of non-common path defocus diversity is introduced which would tend to aggravate the WFS\&C induced anisoplanatism - which is one of the very reasons for using NIRSpec for WFS to begin with. However, translating the NIRCam focal plane has the important advantage of not requiring secondary mirror motion. 
For the purpose of this study we assumed that diversity defocus was introduced by translating the secondary mirror. This was simulated in the MTB setup by utilizing a fixed-lens WFS technique as shown in Figure 4. The lenses were designed and installed into a filter wheel with the defocus values indicated in Figure 4.

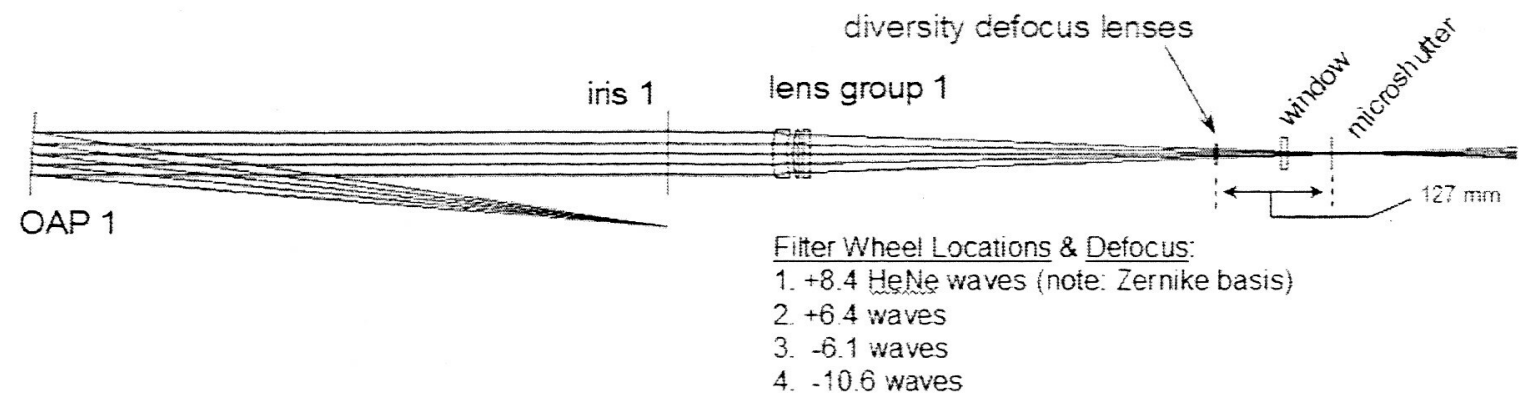

Figure 4. Fixed-Lens WFS Setup for the MTB.

WFS accuracy has been characterized earlier ${ }^{25}$ using this technique and this is also the method of introducing diversity defocus using the JWST NIRCam. The technique also has the advantage simplifying the experimental procedure given that translating the InSb camera (to achieve defocus) is complicated by the bulky nature of its on-board dewar.
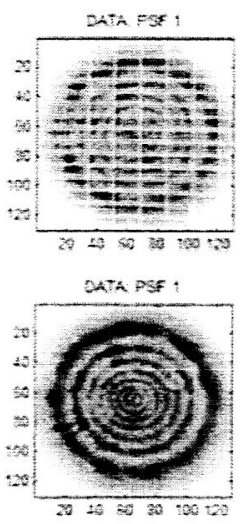
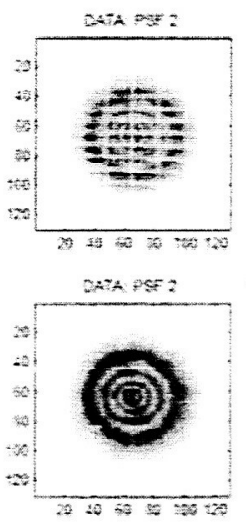
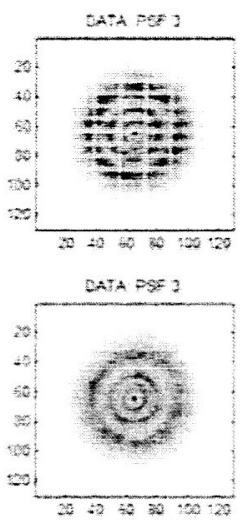
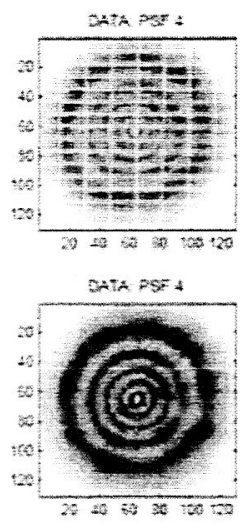

Figure 5. Diversity Defocus Images with and without the MSA light shield.

Sample mages collected at the focal plane of Figure 3 are shown in Figure 5. The top row shows the defocused images formed when the MSA is in place which clearly shows the MSA diffraction structure in the images. The bottom row shows the same defocus setting without the MSA. The sign convention for defocus is indicated at the bottom of Figure 5.

As shown by the top row of Figure 5, the microshutters combine with the PSF complex amplitude and produce diffraction in the Fourier domain of the system stop. Since this image is relayed following the MSA, an additional pupil is formed and therefore a $3^{\text {rd }}$ direction plane exists before the image is formed on the CCD. Therefore, a multi-plane 
diffraction model correctly describes how the images are formed at the detector. This multi-plane diffraction propagation is illustrated graphically in Figure 6.

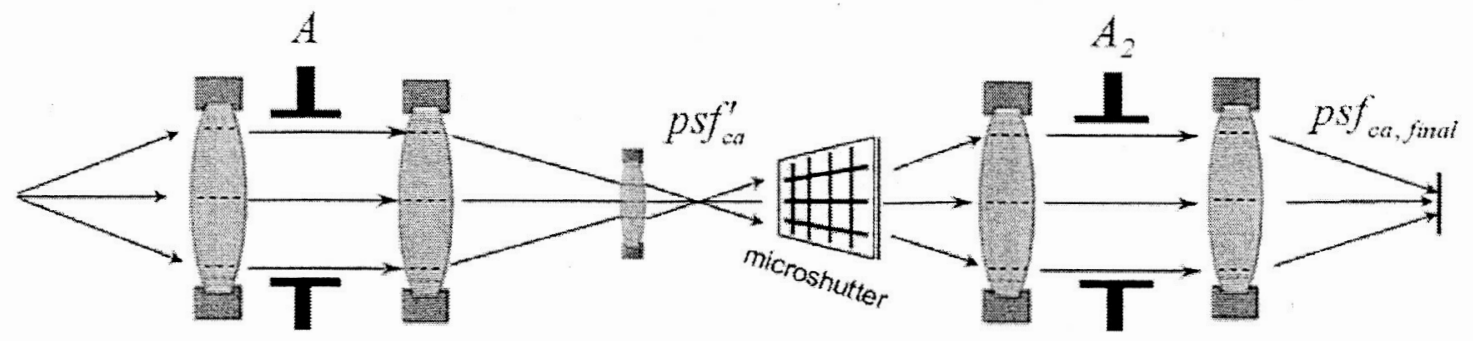

Figure 6. Multi-plane diffraction propagation.

The calculation proceeds by first calculating the complex amplitude of the point spread function (PSF) in a defocused image plane at the microshutter:

$$
p s f_{c a}=\mathfrak{I}\left\{\text { pupil }_{c a}\right\}=\mathfrak{I}\left\{A e^{i k \varphi_{i}}\right\},
$$

where $A$ is a mask defined by the telescope pupil geometry and $\varphi_{d}$ is a defocus phase term (the values used are listed in Figure 4). For JWST the mask A has an 18 segment hexagonal structure (see for instance Ref. ${ }^{12}$ ) but for simplicity in our modeling we assume an un-obscured circular aperture. To properly incorporate diffraction by the microshutter light shield we next multiply the PSF complex amplitude by the microshutter light shield mask

$$
p s f_{c a}^{\prime}=p s f_{c a} \times \text { light shield mask. }
$$

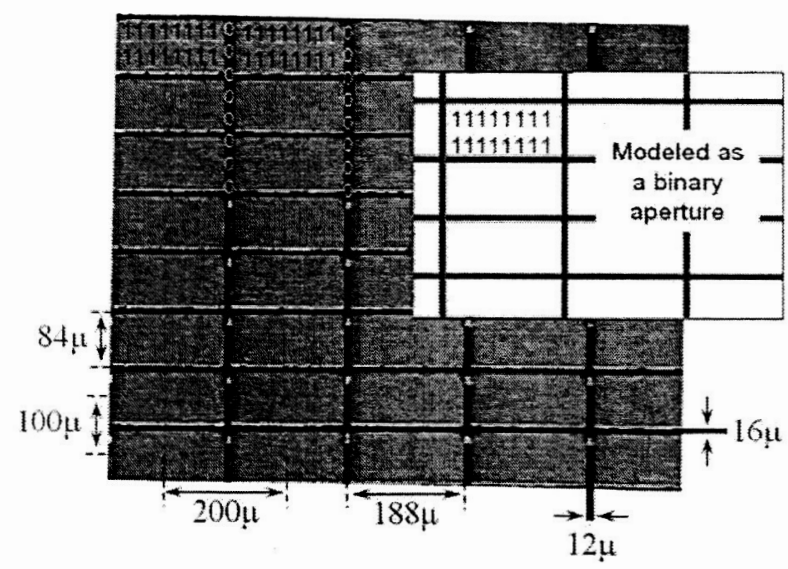

Figure 7. Microshutter Array Light shield.

The light shield mask is defined by an array of zeros and ones $(1 \equiv$ transmission; $0 \equiv$ blocked) as indicated in Figure 7 which shows a microscope image of the light shield as well as its dimensions.

The microshutters are assumed open over the region defined by the defocused image plane. If not, then the defocused images become severely under-sampled by the shutterblocked portion of the images. An example of this is shown in Figure 8 which clearly indicates the consequence of not having all the microshutters open. 


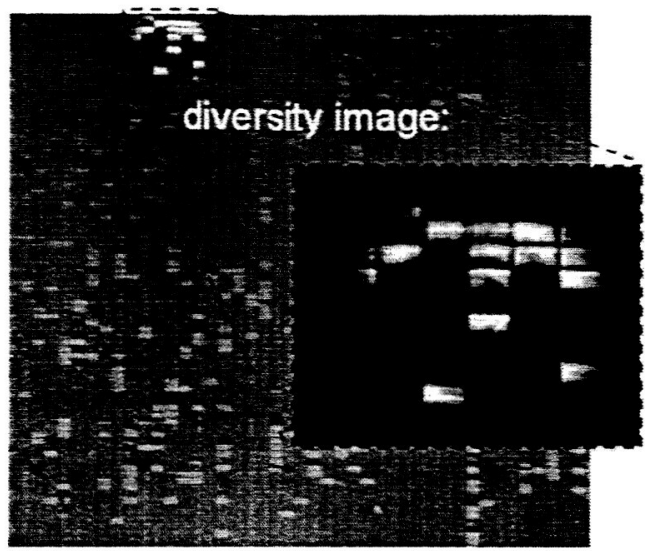

Figure 8. Diversity Defocus Image with un-opened Microshutters.

After propagation to the MSA the beam is then re-collimated and stopped down by $A_{2}$ as shown in Figure 6 . This calculation is modeled as an inverse Fourier transform to the complex pupil plane:

$$
\text { pupil } l_{c a}^{\prime}=\mathfrak{S}^{-1}\left\{p s f_{c a}^{\prime}\right\} \times A_{2},
$$

and then the complex amplitude at the detector is given by:

$$
p s f_{c a, \text { final }}=\mathfrak{S}\left\{\text { pupil }_{c a}^{\prime}\right\} \text {. }
$$

The measured intensity is the complex conjugate square of Equation (4). This calculation describes the multi-plane forward diffraction model illustrated in Figure 6 which is based on the MTB design of Figure 2.

As an application of this approach consider Figure 9 which shows a direct comparison of the data with the forward diffraction model discussed above. The top row is the data (duplicated from Figure 5) and the bottom row are the corresponding calculated images. Clearly, the multi-plane forward model captures the physical effect of diffraction as seen by the diffraction blur the light shield in the of the bottom row of Figure 9 .
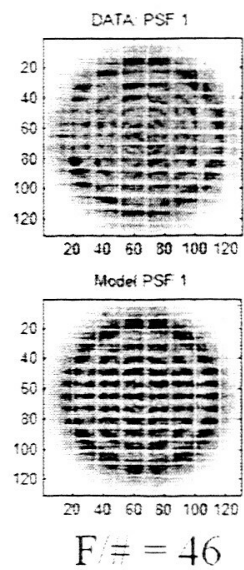
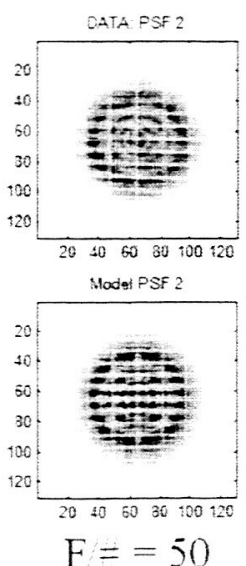

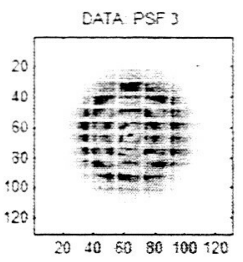

Model PSF 3

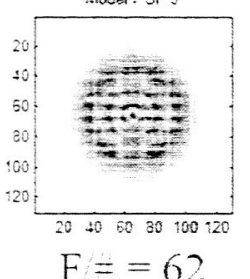

DATA. PSF 4

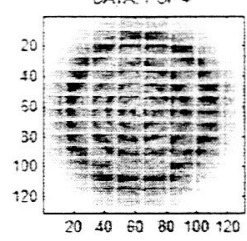

Atodel PSF 4

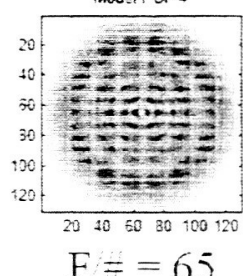

Figure 9. Diversity Defocus Image Data (top row) and Calculated Model Images 
The $\mathrm{F} / \#$ variation with defocus is due to the non-telecentric nature of the experimental setup. The F/\#'s were determined by a fitting procedure ${ }^{13}$ which matches the Fresnel zone structure of the images (concentric circles) with the specified amount of defocus shown in Figure 4. Initially, the F/\#'s or defocus are not assumed to be known exactly but are used as starting values to the fitting procedure. The values shown in Figure 9 and Figure 4 are the final values determined by the fitting procedure.

\section{Image-based WFS using MOS Data}

One goal in this study has been to compare WFS results obtained from each of the data sets shown in Figure 5 to assess the impact on WFS accuracy due to the presence of the MS light shield. A multi-plane diffraction calculation was also examined to model the effect of the MS light shield on the intensity data in a defocused plane. Comparison of the modeled data to the testbed data shows good qualitative agreement as discussed in Section 3. In this Section we motivate the direct application of the image-based WFS algorithm to the data. Therefore, one considers a simple overlay of the MS light shield to the defocused intensity data, i.e., a direct multiplication of the light shield with the PSF intensity data. Therefore, no diffraction effect of the MS is considered since Equation (2) becomes:

$$
p s f^{\prime 2}=p s f^{2} \times \text { light shield mask, }
$$

as seen at the detector. A calculation comparison to the multi-plane model is made in Figure 10.
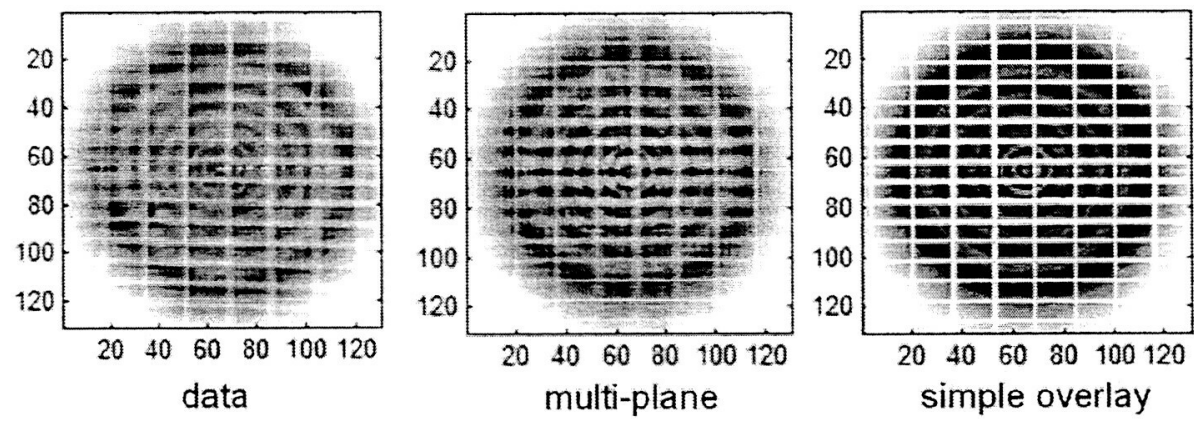

Figure 10. Diversity Defocus Image Data (top row) and Calculated Model Images

The image on the far left is the data. The $2^{\text {nd }}$ image is based on the calculation described in Equations (1) to (4). The far right image is based on the calculation of only (1) and (5) - By comparison of the calculated images with the data above it is apparent that the diffraction effect captured by the multi-plane model is "blur" surrounding the MS light shield mask. However, at least to first order, the calculation described in (5) serves as a good approximation to the multi-plane approach. Physically, the reason this latter approach serves as a reasonable approximation is based on the observation that for a sufficiently defocused image, the image intensity begins to approximate the pupil. As a result, the effect of the MS light shield on the intensity data can be thought of as applying a binary aperture or obscuration at a pupil (the defocused image plane in this case approximates the pupil). As a result, one might expect that reasonably good WFS performance can be achieved without incorporating a multi-plane diffraction model into the forward Fourier propagations of the WFS algorithm. 
A number of image-based phase-retrieval techniques have been developed that can be classified into one of two general categories: (a) iterative-transform ${ }^{14},{ }^{15}$ or (b) parametric. ${ }^{16,17}$ Modifications to the original iterative transform approach have been based on the introduction of a defocus diversity function ${ }^{18,19}$ or on the input-output method $^{20}$. Various implementations of the focus-diverse iterative-transform method have appeared in ${ }^{21}$ deviating slightly by utilizing a single wavelength and varying the placement and number of defocused image planes. Modifications to the parametric approach include minimizing alternative objective functions as well as implementing a variety of nonlinear optimization methods such as Levenburg-Marquardt, simplex, and quasi-Newton techniques. ${ }^{22}$ The current algorithm implementation strategy for JWST is discussed in these proceedings by Acton, et. al. ${ }^{23}$

In this paper, a further variant ${ }^{24,25}$ on the iterative transform method is applied that has the advantages of both the iterative-transform (high-spatial frequency) and parametric (high dynamic range) phase-recoveries. This is achieved by utilizing a "floating" or adaptive diversity function to incorporate feedback during phase-recovery to guide the PR process. A related approach has been discussed by Cohen, et. al. ${ }^{26}$

The first case considered is the data set of the $2^{\text {nd }}$ row in Figure 5, i.e., with no MS in place. The input data set to the WFS algorithm is thus 4 images -2 on each side of best focus. Phase-retrieval results from this data are summarized by the GUI snapshot (graphical user interface) shown in Figure 11 . The results are then decomposed into a Zernike ${ }^{27}$ basis set with dominant components $Z_{6} \approx 0.05$ HeNe waves (astigmatism) and $Z_{13} \approx-0.052$ waves (spherical). The recovered phase is then used to recalculate one of the images of the data set (defocused image \#4) to check consistency. This image is labeled as the MODEL image in Figure 11. $\mathrm{X}$ and $\mathrm{Y}$ cross-sections of the model image are also compared with $\mathrm{X}$ and $\mathrm{Y}$ cross-sections of the data. This latter comparison gives a qualitative consistency check on the assumed F/\# and defocus values. These values can be fine-tuned using the GUI interface and the PR results subsequently updated. 


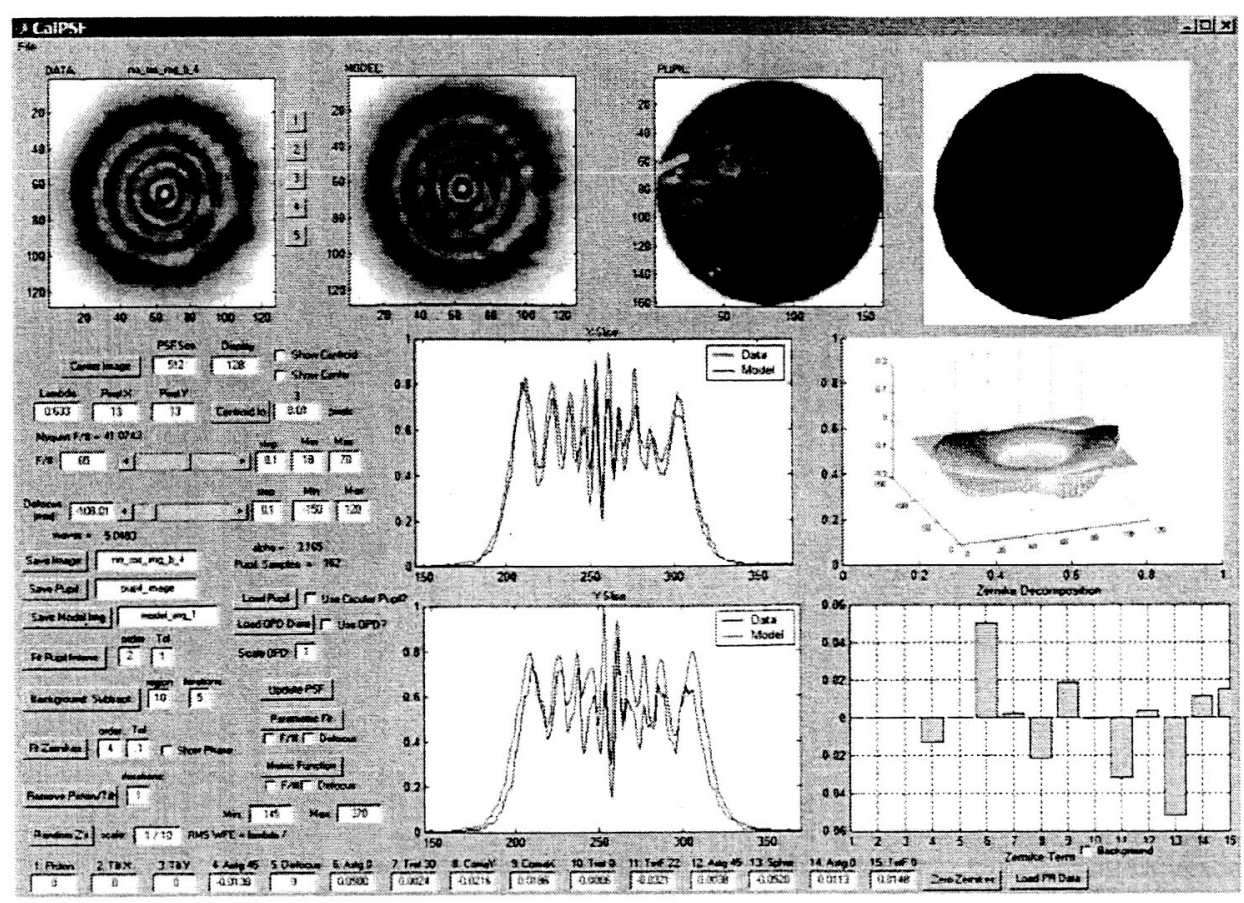

Figure 11. Phase-Retrieval GUI and WFS Results without the Microshutter

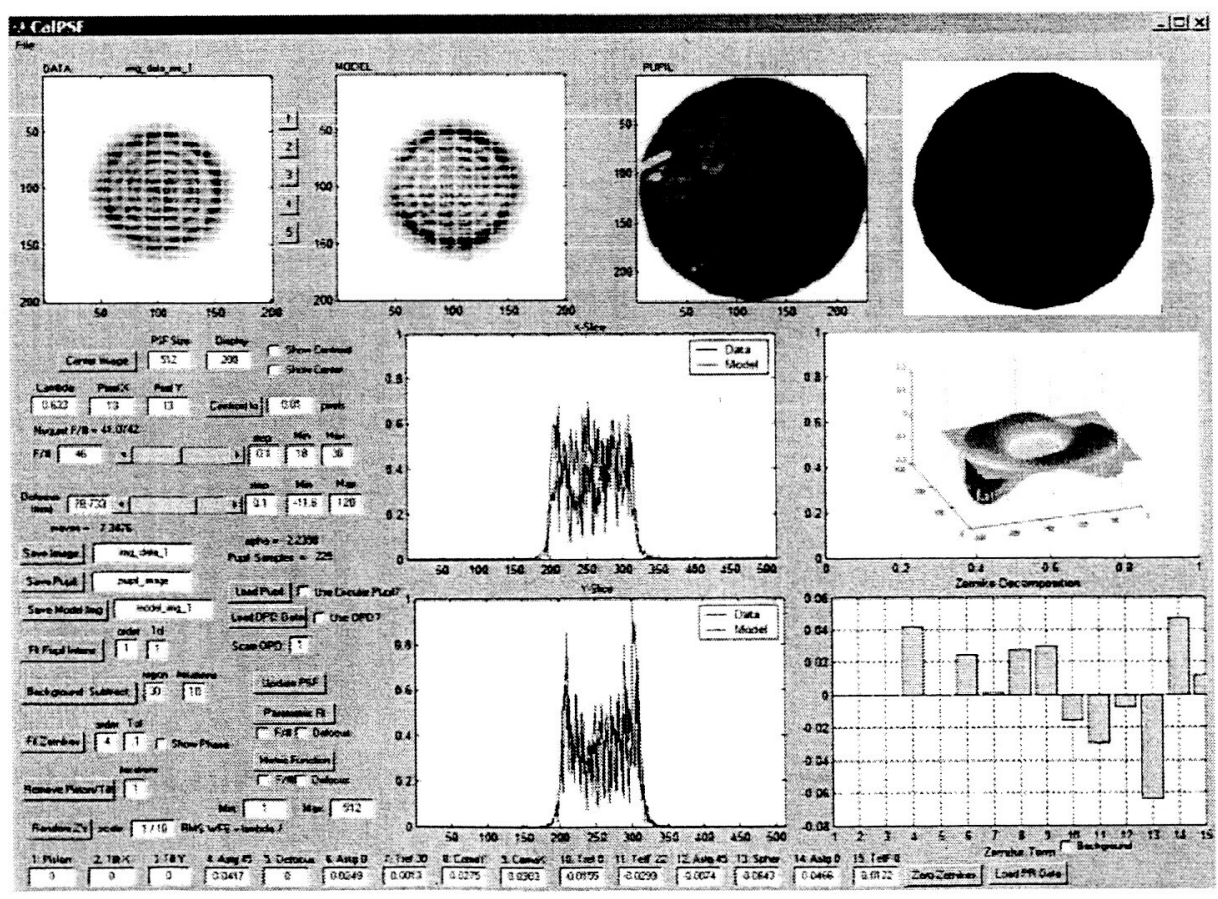

Figure 12. Phase-Retrieval GUI and WFS Results with the Microshutter. 
The second case considered is the data set of the top row in Figure 5, i.e., with the MS in place. Phase-retrieval results from this data are summarized by the GUI image of Figure 12. The results are again decomposed into the Zernike basis set. The MS data PR results differ slightly from the results of Figure 11 with components $Z_{6} \approx 0.025 \mathrm{HeNe}$ waves (astigmatism) and $Z_{13} \approx-0.06$ waves (spherical). The MS data PR results also have a larger component of first order $45 \mathrm{deg}$ astigmatism, $Z_{4}$, as well as second order 0 -degree astigmatism, $Z_{14}$. The recovered phase is again used to recalculate one of the images of the data set to check consistency. Although the PR results vary somewhat between the two cases, the results are still within general agreement given that the data sets were collected on two different days and the system could have been slightly disturbed while adding or removing the MSA. In summary for the low order PR difference between the two cases (with and without the MS) these two results are subtracted as shown in Figure 13. The RMS difference between the two cases is $\approx \lambda / 31$.

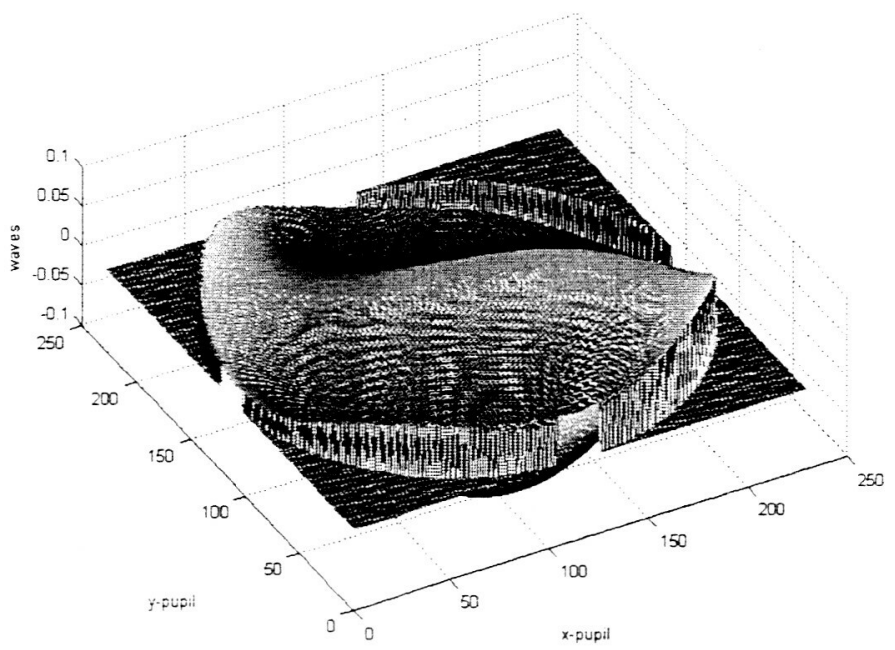

Figure 13. Low-Order PR recovery difference with/without MS .

An alternative comparison examines the PR recovery difference of the higher spatial frequency component by first subtracting the first 15 Zernike terms and then differencing the PR results with/without the MS. The phase difference between the two cases is shown in Figure 14. The RMS value is $\approx \lambda / 15$. The structure of the higher spatial frequency component is better understood by calculating the PSD of the phase difference shown in Figure 14. This result is shown in Figure 15. The "cross-like" structure of the PSD indicates a remnant of the rectangular MS grid. Therefore, the higher spatial frequency component of the MS PR result contains the rectangular grid structure of the MS. As far as WFS and control is concerned, the rectangular component should be filtered to avoid any attempt by the control system to command this pattern onto the primary mirror. This filtering is effectively accomplished by decomposing the PR results into a lower order aberration basis set as indicated in Figure 12. 


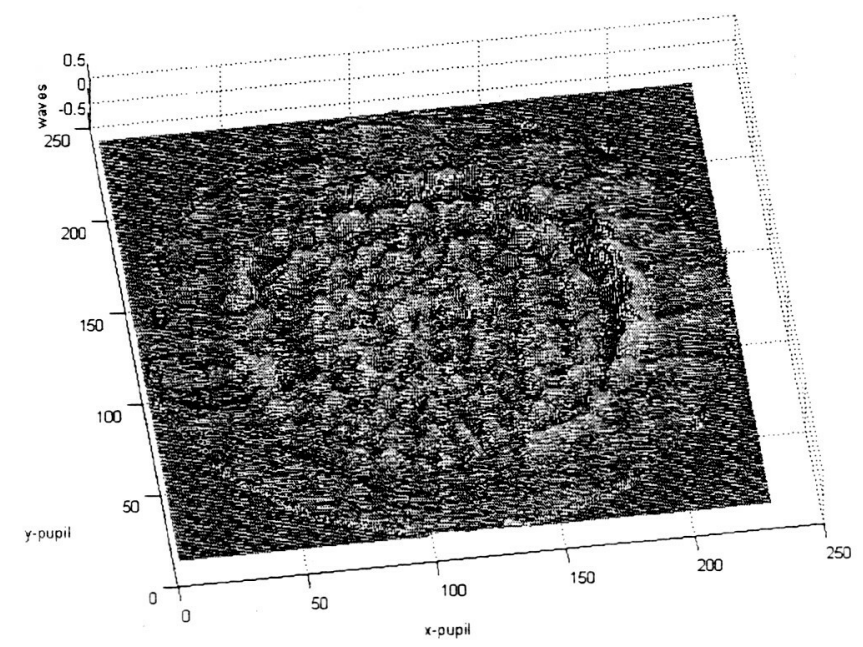

Figure 14. High Spatial Frequency recovery difference with/without MS.

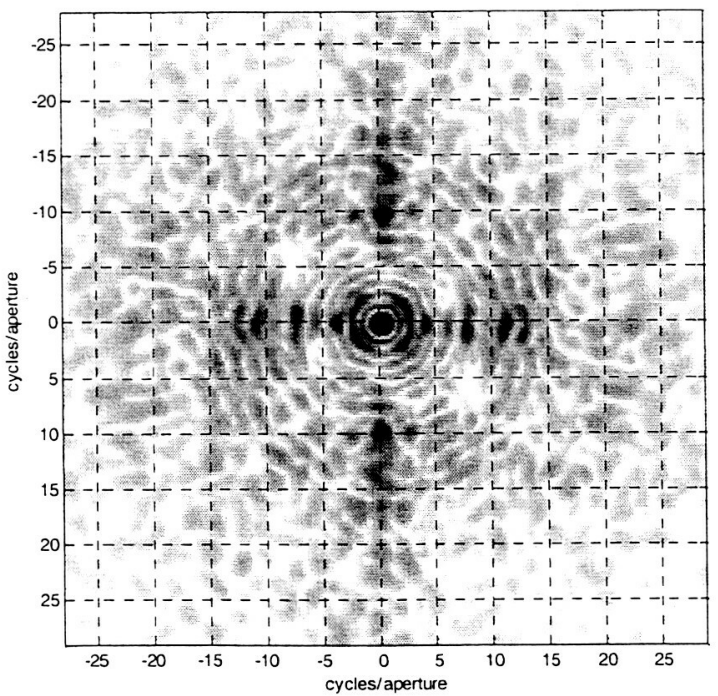

Figure 15. PSD of High Spatial Frequency recovery difference with/without MS . 


\section{Summary and Discussion}

This paper examines PR results from a data set that includes a JWST MS array inserted into the defocused beam path. WFS results were reported on each of the data sets shown in Figure 5 to assess the impact on WFS accuracy due to the presence of the MS light shield. A multi-plane diffraction calculation was also examined to model the effect of the MS light shield on the intensity data in a defocused image plane. Comparison of the modeled data to the testbed data shows good qualitative agreement as discussed in Section 3. We have also shown that consistent PR results can be obtained by considering a direct application of the image-based WFS algorithm ${ }^{24,25}$ to the data without any special modifications to the algorithm procedure.

The MS element behaves as a diffracting element and introduces a rectangular artifact into the recovered phase when compared with PR results obtained from the corresponding data set without the MS. For the data sets considered in this study, the largest difference between the PR results with/without the MS array was due to the higher spatial frequency content. The RMS value of this rectangular artifact was approximately $\lambda / 15$. By contrast, the lower-order PR results (represented by the first 15 Zernike coefficients) show that the PR difference is about a factor of two smaller or $\approx \lambda / 31$.

A basic conclusion from the study is that image-based WFS using NIRSpec MOS data will produce PR results with two dominant components: (a) a low-order component, and (b) a higher spatial frequency component left as a remnant of the MS element. JWST will employ radius of curvature actuator control for each primary mirror segment and therefore the first component (a) will be the only component of the two which can affect the primary mirror figure through the WFS and control system. The higher spatial frequency component can be effectively filtered out by decomposing the PR results into a lower order aberration basis set as indicated in Figure 12.

Additional work is underway which considers the effect of the JWST aperture on the diversity defocus data + the MS element. I.e., one effect of the MS light shield is to slightly under-sample the data since some pixels are blocked in the data collection. The effect of this on segmented aperture recoveries is unknown.

Another important effect not fully examined in this paper deals with the rather poor SNR of data collected by the MTB InSb camera. Many hundreds of frames must co-added to obtain narrow-band images of sufficient SNR to perform accurate WFS. For example, Figure 16 shows the InSb analog of the top row of Figure 5. The pixel size of the InSb camera is $30 \mu$ square and therefore the MS light shield rectangular regions appear much smaller. Each image represents the sum of 100 broadband images after background subtraction and flat fielding. 

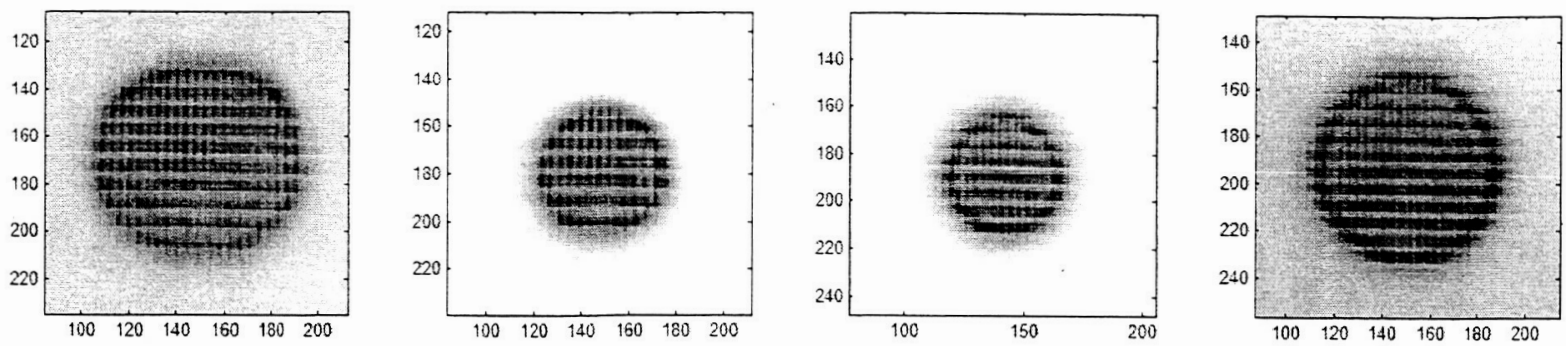

Figure 16. Broadband InSb Diversity Defocus Data Sets $(\lambda \in 1$ to $5 \mu)$.

Additionally, detector effects such as charge diffusion tend to be more pronounced using IR detectors and will likely further degrade WFS accuracy. Under such conditions the RMS differences between WFS results with/without the MS element could be substantially larger. The combination of blurring due to detector effects such as charge diffusion in addition to the inherent blur of broadband data will impact WFS accuracy 28 but generally only the higher spatial frequency component will be significantly impacted. Experiments are currently underway to obtain sufficient SNR in the IR image data utilizing a narrowband $2 \mu$ filter. Wavefront sensing error as a function of these factors will be reported in a future study.

\section{Acknowledgements}

The research described in this paper was carried out at the NASA GSFC. The authors acknowledge support from the JWST project office as well as helpful discussion with Lee Feinberg, Bill Hayden, Scott Acton, Paul Atcheson, and Dave Redding.

\section{References}

1. http://jwst.gsfc.nasa.gov/

2. http://origins.jpl.nasa.gov

3. http://www.stsci.edu/jwst/instruments/nirspec/

4. Northrop Grumman has chosen the beryllium-based mirror technology made by Ball Aerospace \& Technologies Corporation as the primary mirror material for JWST.

5. http://ircamera.as.arizona.edu/nircam/

6.

7.

8. S.H. Moseley, K.A. Blumenstock, et.al., "Microshutter arrays for JWST - Programmable Field Masks,” 201 st Meeting of the American Astronomical Society (AAS) Seattle WA, January 2003. 
A. S. Kutyrev, R. Arendt, S. H. Moseley, R. A. Boucarut, T. Hadjimichael, M. Jhabvala, T. King, M. Li, J. Loughlin, D. Rapchun, D. S. Schwinger and R. F. Silverberg, "Programmable microshutter arrays for the JWST NIRSpec", accepted for publication in the Journal on Selected Topics in Quantum Electronics on Optical Microsystems, April 2004.

Kutyrev, A.S.; Moseley, S.H.; Boucarut, R.A.; Jhabvala, M.; Li, M.; Schwhiger, D.S.; Silverberg, R.F.; Wesenberg, R.P.; Programmable microshutter arrays for the JWST NIRSpec, Optical MEMS IEEE/LEOS International Conference on, 18-21 Aug. 2003, pp. 87 - 88

9. J.W. MacKenty, K. Ray, M.A. Greenhouse, R.G. Ohl, H.A. Knutson, R.F. Green, IRMOS Team, "Integration and Testing of IRMOS," AAS 203rd Meeting, Observations and Instrumentation, January 6, 2004

http://www.stsci.edu/projects/IRMOS/

10. R. Lyon and R. Arendt.

11. B. H. Dean \& C W. Bowers, "Diversity selection for phase-diverse phase retrieval", J. Opt. Soc. Am. A., vol 20, pp. 1490-1504, August 2002.

12. J. J. Green, B. H. Dean, Catherine M. Ohara, Yan Zhang, "Target selection and imaging requirements for JWST fine phasing," Proc. SPIE, 5487 - these proceedings (2004).

13. B.H. Dean, "Wavefront Control Testbed PSF Magnifier Data," NASA GSFC, Oct. 6 (2000).

14. R.W. Gerchberg and W.O. Saxton, "Phase Determination from Image and Diffraction Plane Pictures in an Electron-Microscope," OPTIK, 34, 275 (1971).

R.W. Gerchberg and W.O. Saxton, "A Practical Algorithm for the Determination of Phase from Image and Diffraction Plane Pictures," OPTIK, 35, 237-246 (1972).

W.O. Saxton, "Computer Techniques for Image Processing in Electron Microscopy," in Advances in Electronics and Electron Physics, Supplement 10, L Marton and C. Marton, eds. (Academic Press, New York, NY, 1978).

15. N. C. Gallagher, B. Liu, "Method for Computing Kineforms that Reduces Image-Reconstruction Error," Appl. Optics 12(10) 2328-2335 (1973)

N. C. Gallagher, B. Liu, "Method for Computing Kineforms that Reduces Image-Reconstruction Error," Appl. Optics 12(10) 2328-2335 (1973)

R. A. Gonsalves, R. Dumais, P. Considine, "On Optimal Holographic Filters," Proc. SPIE, Vol. $45,1974$.

16. R. A. Gonsalves, P. Considine, "Phase-Retrieval from Modulus Data," J. Opt. Soc. Am., 66, 961964 (1976).

17. W. H. Southwell, "Wavefront Analyzer using a Maximum Likelihood Algorithm," J. Opt. Soc. Am. A3, 396-399 (1977).

18. D.L. Misell, "A method for the solution of the phase problem in electron microscopy," J Phys., D6, L6-L9 (1973).

19. R. A. Gonsalves, "Phase Retrieval and Diversity in Adaptive Optics", Optical Engineering, 21(5), 829-832, (1982). 
20. J.R. Fienup, “ ,’J. Opt. Soc. Am. 64, 1395A (1974).

J.R. Fienup, “Phase Retrieval Algorithms: a Comparison,” Appl Opt., 21(15), 2758-2769, (1982).

21 C. Roddier, F. Roddier, "Wavefront Reconstruction from Defocused Images and the Testing of Ground-based Optical Telescopes," J. Opt. Soc. Am., A10, 2277-2287 (1993).

C. Roddier, F. Roddier, "Combined Approach to the Hubble Space Telescope Wave-Front Distortion Analysis," Appl. Opt., 32, 2992-3008 (1993).

J.R. Fienup, J.C. Marron, R. G. Paxman, T.J. Schulz, J. H. Seldin, B. Thelen, "Image Inversion Analysis of the Hubble Space Telescope," in Final Report: JPL Contract 958892 on HST OTA Analysis, August 1991.

R. G. Lyon, "DCATT Comparative Wavefront Sensing and Optical Control Study Final Report," Feb. 22, 1999. http://jansky.gsfc.nasa.gov/OSCAR/

D. C. Redding, et al, "Next Generation Space Telescope wavefront sensing and control", Proc. SPIE, 4850 (2002).

22. William H. Press, Brian P. Flannery, Saul A. Teukolsky, William T. Vetterling, "Numerical Recipes: The Art of Scientific Computing," Cambridge University Press.

23. S. Acton, et al, Proc. SPIE, 5487 - these proceedings (2004).

24. B. H. Dean "Phase Retrieval for Extended Dynamic Range: Test Results using Low-Order Aberration Cases," NASA GSFC, Jan 2001.

B. H. Dean "Phase-Retrieval Analysis of Hubble Simulator Data," NASA GSFC, October 1, 2002.

B. H. Dean "Preliminary Phase-Retrieval Assessment of MLA Images," NASA GSFC, April 9, 2003.

25. B. H. Dean "Results on Fixed Lens Wavefront Sensing," presented at the JWST Technical Memorandum Discussion, NASA GSFC, June 5, 2003

26. D. Cohen and D. C. Redding, "NGST high dynamic range unwrapped phase estimation," Proc. SPIE, 4850 (2002).

27. D. Malacara, S DeVore, "Interferogram Evaluation and Wavefront Fitting," in Optical Shop Testing, D. Malacara, ed., (Wiley-Interscience, NewYork, NY, 1997).

28. B. H. Dean, "Cramer-Rao Analysis for Phase-Diverse-Phase-Retrieval: Diversity Functions and Broadband Phase-Retrieval," presented at the Wavefront Sensing \& Controls Conference, sponsored by Kamuela Optical Associates, Hawaii, Nov. 13-16, 2000. 